\title{
New Iterative Methods for Solving Fokker-Planck Equation
}

\author{
A. A. Hemeda $\mathbb{D}$ and E. E. Eladdad \\ Department of Mathematics, Faculty of Science, Tanta University, Tanta 31527, Egypt
}

Correspondence should be addressed to A. A. Hemeda; aahemeda@yahoo.com

Received 18 July 2018; Revised 25 October 2018; Accepted 15 November 2018; Published 28 November 2018

Academic Editor: Maria L. Gandarias

Copyright (c) 2018 A. A. Hemeda and E. E. Eladdad. This is an open access article distributed under the Creative Commons Attribution License, which permits unrestricted use, distribution, and reproduction in any medium, provided the original work is properly cited.

In this article, we propose the new iterative method and introduce the integral iterative method to solve linear and nonlinear Fokker-Planck equations and some similar equations. The results obtained by the two methods are compared with those obtained by both Adomian decomposition and variational iteration methods. Comparison shows that the two methods are more effective and convenient to use and overcome the difficulties arising in calculating Adomian polynomials and Lagrange multipliers, which means that the considered methods can simply and successfully be applied to a large class of problems.

\section{Introduction}

In the last years, many of the new methods were used for solving linear and nonlinear functional equations arising from the mathematical\} modeling of problems in mathematics, physics, engineering, and other different science branches which have important applications in life such as Adomian decomposition method (ADM) [1, 2], variational iteration method (VIM) [3-7], homotopy perturbation method (HPM) [8-13], homotopy analysis method (HAM) $[8,14]$, new iterative method (NIM) [15-21], differential transform method (DTM) [22], Laplace transform method (LTM) [1, 22], integral iterative method (IIM) [23, 24], and other methods [25-29].

NIM, proposed first by Daftardar-Gejji and Jafari in 2006 [15-21], is used simply and accurately for solving a variety of linear and nonlinear equations such as algebraic equations, integral equations, integrodifferential equations, ordinary and partial differential equations of integer and fractional order, and systems of equations as well.

IIM $[23,24]$ is a new iterative method based explicitly on the integral operator; the inverse of the differential operator in the problem under consideration, this method is an accurate and reliable method, which can be used simply and accurately to a large class of problems better than the NIM, $\mathrm{ADM}$, and VIM as will be seen in Applications.
These two methods are very convenient, effective, and accurate. The basic ideas of the two methods are considered in Section 3.

Fokker-Planck equation (FPE), first applied to investigate the Brownian motion of particles, is now largely employed in various generalized forms, in physics, engineering biology, and chemistry.

The motivation of this work is to extend the application of the NIM and IIM for solving linear and nonlinear FPEs and comparing the results obtained by these methods with those obtained by ADM and VIM. In [2, 7], ADM, VIM, and HPM were applied to solve FPE.

\section{Fokker-Planck Equation (FPE)}

The general form of FPE for variables $x$ and $t$ is as follows $[2,7,13,28,29]$ :

$$
\frac{\partial u}{\partial t}=\left[-\frac{\partial}{\partial x} A(x)+\frac{\partial^{2}}{\partial x^{2}} B(x)\right] u(x, t),
$$

with the initial condition

$$
u(x, 0)=f(x), \quad x \in \operatorname{Re},
$$


where $A(x)$ is the drift coefficient and $B(x)>0$ is the diffusion coefficient. The drift and diffusion coefficients can also be functions of $x$ and $t$; that is,

$$
\frac{\partial u}{\partial t}=\left[-\frac{\partial}{\partial x} A(x, t)+\frac{\partial^{2}}{\partial x^{2}} B(x, t)\right] u(x, t) .
$$

Equation (1) is a linear second-order partial differential equation of parabolic type, called Kolmogorov equation, which is an equation for the motion of a concentration field $u(x, t)$. The backward Kolmogorov equation can be written in the following form:

$$
\frac{\partial u}{\partial t}=-\left[A(x, t) \frac{\partial}{\partial x}+B(x, t) \frac{\partial^{2}}{\partial x^{2}}\right] u(x, t) .
$$

A generalized form of (1) to $N$ variables $x_{1}, \ldots, x_{N}$ can be written as follows:

$$
\frac{\partial u}{\partial t}=\left[-\sum_{i=1}^{N} \frac{\partial}{\partial x_{i}} A_{i}(x)+\sum_{i, j=1}^{N} \frac{\partial^{2}}{\partial x_{i} \partial x_{j}} B_{i, j}(x)\right] u(x, t),
$$

with the initial condition

$$
u(x, 0)=f(x), \quad x=\left(x_{1}, x_{2}, \ldots, x_{N}\right) \in R^{N},
$$

where the drift vector $A_{i}$ and the diffusion tensor $B_{i, j}$ in (5) depend on $N$ variables $x_{1}, \ldots, x_{N}$.

There is a more general form of FPE, which is nonlinear FPE. Nonlinear FPE has important applications in various areas such as plasma physics, surface physics, population dynamics, biophysics, engineering, neurosciences, nonlinear hydrodynamics, polymer physics, laser physics, pattern formation, psychology, and marketing. The nonlinear FPE for one variable is in the following form:

$$
\frac{\partial u}{\partial t}=\left[-\frac{\partial}{\partial x} A(x, t, u)+\frac{\partial^{2}}{\partial x^{2}} B(x, t, u)\right] u(x, t) .
$$
form:

Equation (7) for $N$ variables $x_{1}, \ldots, x_{N}$ is in the following

$$
\begin{gathered}
\frac{\partial u}{\partial t}=\left[-\sum_{i=1}^{N} \frac{\partial}{\partial x_{i}} A_{i}(x, t, u)+\sum_{i, j=1}^{N} \frac{\partial^{2}}{\partial x_{i} \partial x_{j}} B_{i, j}(x, t, u)\right] \\
\cdot u(x, t), \quad x=\left(x_{1}, x_{2}, \ldots, x_{N}\right) \in R^{N} .
\end{gathered}
$$

\section{Analysis of the Methods}

In this section, we introduce the analysis of the two considered methods.

3.1. New Iterative Method (NIM). To illustrate the basic idea of NIM, we consider the following general functional equation [15-21]:

$$
u(x)=f(x)+N(u(x)),
$$

where $N$ is a nonlinear operator from a Banach space $B \longrightarrow B$ and $f$ is a known function (element) of the Banach space $B$. We are looking for a solution $u(x)$ of (9) having the series form:

$$
u(x)=\sum_{i=0}^{\infty} u_{i}(x)
$$

The nonlinear operator $N$ can be decomposed as

$$
\begin{aligned}
N\left(\sum_{i=0}^{\infty} u_{i}\right)= & N\left(u_{0}\right) \\
& +\sum_{i=1}^{\infty}\left\{N\left(\sum_{j=0}^{i} u_{j}\right)-N\left(\sum_{j=0}^{i-1} u_{j}\right)\right\} .
\end{aligned}
$$

From (10) and (11), (9) is equivalent to

$$
\begin{aligned}
\sum_{i=0}^{\infty} u_{i}= & +N\left(u_{0}\right) \\
& +\sum_{i=1}^{\infty}\left\{N\left(\sum_{j=0}^{i} u_{j}\right)-N\left(\sum_{j=0}^{i-1} u_{j}\right)\right\} .
\end{aligned}
$$

The required solution $u(x)$ for (9) can be obtained recurrencely from the recurrence relation:

$$
\begin{aligned}
u_{0}= & f, \\
u_{1}= & N\left(u_{0}\right), \\
u_{r+1}= & N\left(u_{0}+u_{1}+\cdots+u_{r}\right) \\
& -N\left(u_{0}+u_{1}+\cdots+u_{r-1}\right),
\end{aligned}
$$

Then

$$
\begin{array}{r}
\left(u_{1}+u_{2}+\cdots+u_{r+1}\right)=N\left(u_{0}+u_{1}+\cdots+u_{r}\right), \\
r=1,2, \ldots
\end{array}
$$

and

$$
\sum_{i=0}^{\infty} u_{i}=f+N\left(\sum_{i=0}^{\infty} u_{i}\right)
$$

The $r$-term approximate solution of (9) and (10) is given by $u(x)=\sum_{i=0}^{r-1} u_{i}$.

Remark 1. If $N$ is a contraction, that is, $\|N(x)-N(y)\| \leq k \| x-$ $y \|, 0<k<1$, then

$$
\left\|u_{r+1}\right\| \leq k^{r+1}\left\|u_{0}\right\|, \quad r=0,1,2, \ldots
$$


Proof. From (13), we have

$$
\begin{aligned}
& u_{0}=f \text {, } \\
& \left\|u_{1}\right\|=\left\|N\left(u_{0}\right)\right\| \leq k\left\|u_{0}\right\|, \\
& \left\|u_{2}\right\|=\left\|N\left(u_{0}+u_{1}\right)-N\left(u_{0}\right)\right\| \leq k\left\|u_{1}\right\| \leq k^{2}\left\|u_{0}\right\|, \\
& \left\|u_{3}\right\|=\left\|N\left(u_{0}+u_{1}+u_{2}\right)-N\left(u_{0}+u_{1}\right)\right\| \leq k\left\|u_{2}\right\| \\
& \leq k^{3}\left\|u_{0}\right\| \\
& \left\|u_{r+1}\right\|=\left\|N\left(u_{0}+\cdots+u_{r}\right)-N\left(u_{0}+\cdots+u_{r-1}\right)\right\| \\
& \leq k\left\|u_{r}\right\| \leq k^{r+1}\left\|u_{0}\right\|,
\end{aligned}
$$

$r=0,1,2, \ldots$, and the series $\sum_{i=0}^{\infty} u_{i}$ absolutely and uniformly converges to a solution of (9) [30], which is unique in view of the Banach fixed-point theorem [31].

3.2. Solving Differential Equations Using NIM. After the above presentation of the NIM, we present a reliable approach of this method. To illustrate the basic idea of the new algorithm, we consider the general partial differential equation of arbitrary order:

$$
\begin{gathered}
\frac{\partial^{n} u(x, t)}{\partial t^{n}}=A(u, \partial u)+B(x, t), \quad n \in N, \\
\frac{\partial^{k} u(x, 0)}{\partial t^{k}}=h_{k}(x), \quad k=0,1,2, \ldots, n-1,
\end{gathered}
$$

where $A$ is a nonlinear function of $u, \partial u$ (partial derivatives of $u$ with respect to $x$ and $t$ ) and $B$ is the source function. In view of the integral operators, the initial value problem in (18) and (19) is equivalent to the following integral equation:

$$
u(x, t)=\sum_{k=0}^{n-1} h_{k}(x) \frac{t^{k}}{k !}+I_{t}^{n} B+I_{t}^{n} A=f+N(u),
$$

where $f=\sum_{k=0}^{n-1} h_{k}(x)\left(t^{k} / k !\right)+I_{t}^{n} B, N(u)=I_{t}^{n} A$, and $I_{t}^{n}$ is an $n$ th-order ( $n$-fold) integral operator.

Remark 2. When the general functional equation (9) is linear, that is, $N$ is a linear operator, the recurrence relation (13) can be simplified in the following form:

$$
\begin{aligned}
u_{0} & =f \\
u_{r+1} & =N\left(u_{r}\right), \quad r=0,1,2, \ldots
\end{aligned}
$$

The required solution $u(x, t)$ for (20) and hence for (18) and (19) can be obtained recurrently by employing the recurrence relation (13) or (21).

3.3. Integral Iterative Method (IIM). The IIM is a new iterative method that depends explicitly on the integral operator, the inverse of the differential operator in the problem under consideration $[23,24]$. To illustrate the basic idea of this method, we consider again the general partial differential equation (18)-(19). Also, in view of the integral operators, the initial value problem (18)-(19) is equivalent to the integral equation (20). The required solution $u(x, t)$ for $(20)$ and hence for (18) and (19) can be obtained recurrently by employing the simple recurrence relation:

$$
\begin{aligned}
u_{0} & =f, \\
u_{r+1} & =u_{0}+N\left(u_{r}\right), \quad r=0,1,2, \ldots,
\end{aligned}
$$

in place of recurrence relation (13) or recurrence relation (21), where $u(x, t)=\lim _{r \rightarrow \infty} u_{r}(x, t)$.

Recurrence relation (22) is the recurrence relation for IIM $[23,24,32]$. The IIM may be considered as a new approach for Picard method (PM), where in PM the terms $f$ and $N(u)$ in (20) take the forms $f=\sum_{k=0}^{n-1} h_{k}(x)\left(t^{k} / k !\right)$ and $N(u)=I_{t}^{n}[A+$ $B]$. By this change, the $r$-order term approximate solution for (18)-(19) by IIM is the same $r$-term approximate solution for it by NIM but without calculating the values: $N\left(\sum_{i=0}^{r} u_{i}\right)-$ $N\left(\sum_{i=0}^{r-1} u_{i}\right), r=1,2, \ldots$ (see $\left.(13)\right)$ or the $r$-term approximate solution from the relation $u(x, t)=\sum_{i=0}^{r-1} u_{i}, r=1,2, \ldots$, which simplify and reduce the computational procedures and time. Also, by this change, there is no need to calculate the integral of the source function $B(x, t)$ for every iteration $r, r=1,2, \ldots$ as done in PM, which also simplifies and reduces the computational procedures and time. In addition to the above advantages of IIM over both NIM and PM, there are more advantages of both IIM and NIM over the other methods which are the fact that there is no need to calculate Adomian's polynomials as done in ADM or the value of Lagrange's multiplier as done in VIM or to equate the terms of equal powers of the embedding parameter $p$ as done in HPM and HAM. Moreover, the considered methods can be used successfully without linearization or small perturbation as done in the perturbation methods. Therefore, IIM is better and easier than NIM, PM, and IIM; NIM is better and easier than the other methods.

The main advantage of the considered methods, as we will see in the next section, is that they can be applicable simply and accurately to a large class of partial differential equations.

\section{Applications}

To illustrate the reliability of the considered methods, six important cases of the FPE will be investigated.

\subsection{Example 1. Consider (1) with the initial condition}

$$
u(x, 0)=x, \quad x \in \operatorname{Re} .
$$

Let in (1) $A(x)=-1, B(x)=1$.

By the NIM, according to (20), the considered initial value problem is equivalent to the integral equation:

$$
u(x, t)=x+I_{t}\left[\left(-\frac{\partial}{\partial x} A(x)+\frac{\partial^{2}}{\partial x^{2}} B(x)\right) u(x, t)\right] .
$$


Let

$$
N(u)=I_{t}\left[\left(-\frac{\partial}{\partial x} A(x)+\frac{\partial^{2}}{\partial x^{2}} B(x)\right) u(x, t)\right] .
$$

In view of (13) or (21), we have the following first few components of the new iterative solution for the considered problem:

$$
\begin{aligned}
& u_{0}(x, t)=x, \\
& u_{1}(x, t)=t, \\
& u_{j}(x, t)=0, \quad j \geq 2 .
\end{aligned}
$$

The $(r+1)$-term new iterative approximate solution for this problem is given by

$$
u(x, t)=\sum_{i=0}^{r} u_{i}(x, t)=x+t,
$$

which is the exact solution.

By the IIM, according to (20) and the initial value problem (1), (23) is equivalent to the integral equation:

$$
\begin{aligned}
u_{r+1}(x, t) & \\
= & u_{0}(x, t) \\
\qquad & +I_{t}\left[\left(-\frac{\partial}{\partial x} A(x)+\frac{\partial^{2}}{\partial x^{2}} B(x)\right) u_{r}(x, t)\right], \\
r & \quad \\
r & \geq 0 .
\end{aligned}
$$

Therefore, from (22), we can obtain the following first few components of the integral iterative solution for (1) and (23):

$$
\begin{aligned}
& u_{0}(x, t)=x, \\
& u_{1}(x, t)=x+t, \\
& u_{r}(x, t)=0, \quad r \geq 2 .
\end{aligned}
$$

Hence,

$$
u(x, t)=\lim _{r \rightarrow \infty} u_{r}(x, t)=x+t,
$$

which is the same result as that obtained by NIM in (27) but without calculating $N\left(\sum_{i=0}^{r} u_{i}\right)-N\left(\sum_{i=0}^{r-1} u_{i}\right), r=1,2, \ldots$, and the $r$-term approximate solution: $u(x)=\sum_{i=0}^{r-1} u_{i}$, which simplifies and reduces the computational procedures and hence reduces the computational time. Therefore, IIM is easier than NIM.

It is clear that the above results obtained by the NIM and IIM in (27) and (30) were obtained by ADM and VIM in [2,7] but without calculating Adomian polynomials and Lagrange multiplier value, which means that these methods simplify and reduce the computational procedures and hence reduce the computational time more than those methods. Therefore, NIM and IIM are better than ADM and VIM.
4.2. Example 2. Consider (3) with the initial condition

$$
u(x, 0)=\sinh x \text {. }
$$

Let in (3)

$$
\begin{aligned}
& A(x, t)=e^{t}[\operatorname{coth} x \cosh x+\sinh x]-\operatorname{coth} x, \\
& B(x, t)=e^{t} \cosh x .
\end{aligned}
$$

By the NIM, according to (20), the considered initial value problem is equivalent to the integral equation:

$$
\begin{aligned}
& u(x, t) \\
& \quad=\sinh x \\
& \quad+I_{t}\left[\left(-\frac{\partial}{\partial x} A(x, t)+\frac{\partial^{2}}{\partial x^{2}} B(x, t)\right) u(x, t)\right] .
\end{aligned}
$$

Let

$$
N(u)=I_{t}\left[\left(-\frac{\partial}{\partial x} A(x, t)+\frac{\partial^{2}}{\partial x^{2}} B(x, t)\right) u(x, t)\right] .
$$

In view of (13) or (21), we have the following first few components of the new iterative solution for the considered problem:

$$
\begin{gathered}
u_{0}(x, t)=\sinh x, \\
u_{1}(x, t)=\sinh x \cdot t, \\
u_{2}(x, t)=\sinh x \cdot \frac{t^{2}}{2}, \\
u_{3}(x, t)=\sinh x \cdot \frac{t^{3}}{6},
\end{gathered}
$$

and so on. In the same manner, the rest of components can be obtained. The $(r+1)$-term approximate solution for this problem, in series form, is given by

$$
u(x, t)=\sum_{i=0}^{r} u_{i}(x, t)
$$

$$
=\sinh x \cdot\left(1+t+\frac{t^{2}}{2 !}+\frac{t^{3}}{3 !}+\cdots+\frac{t^{r}}{r !}\right),
$$

which in closed form is equivalent to the exact solution:

$$
u(x, t)=e^{t} \sinh x .
$$

By the IIM, according to (20), the initial value problem ((3) and (31)) is equivalent to the integral equation:

$$
\begin{aligned}
u_{r+1}(x, t) & \\
=u_{0}(x, t) & \\
+I_{t}\left[\left(-\frac{\partial}{\partial x} A(x, t)+\frac{\partial^{2}}{\partial x^{2}} B(x, t)\right) u_{r}(x, t)\right], & \\
r & \geq 0 .
\end{aligned}
$$


Therefore, from (22), we can obtain the following first few components of the integral iterative solution for (3) and (31):

$$
\begin{gathered}
u_{0}(x, t)=\sinh x, \\
u_{1}(x, t)=\sinh x \cdot(1+t), \\
u_{2}(x, t)=\sinh x \cdot\left(1+t+\frac{t^{2}}{2}\right), \\
u_{3}(x, t)=\sinh x \cdot\left(1+t+\frac{t^{2}}{2}+\frac{t^{3}}{6}\right),
\end{gathered}
$$

and so on. In the same manner, the rest of components can be obtained. Hence,

$$
u_{r}(x, t)=\sinh x \cdot\left(1+t+\frac{t^{2}}{2 !}+\frac{t^{3}}{3 !}+\cdots+\frac{t^{r}}{r !}\right) .
$$

In closed form, this gives

$$
u(x, t)=\lim _{r \longrightarrow \infty} u_{r}=e^{t} \sinh x
$$

which is the same result as that obtained by NIM in (37) but without calculating $N\left(\sum_{i=0}^{r} u_{i}\right)-N\left(\sum_{i=0}^{r-1} u_{i}\right), r=1,2, \ldots$, and $u(x)=\sum_{i=0}^{r-1} u_{i}$. Also, it is the same result as that obtained by ADM and VIM in [2,7] but without calculating Adomian polynomials and Lagrange multiplier value. Therefore, IIM is easier than NIM and NIM; IIM is better than ADM and VIM.

4.3. Example 3. Consider the backward Kolmogorov equation (4) with the initial condition:

$$
u(x, 0)=1+x, \quad x \in \operatorname{Re} .
$$

Also, let in (4)

$$
\begin{aligned}
& A(x, t)=-(1+x), \\
& B(x, t)=x^{2} e^{t} .
\end{aligned}
$$

By the NIM, according to (20), the considered initial value problem is equivalent to the integral equation:

$$
\begin{aligned}
& u(x, t) \\
& =1+x \\
& \quad-I_{t}\left[\left(A(x, t) \frac{\partial}{\partial x}+B(x, t) \frac{\partial^{2}}{\partial x^{2}}\right) u(x, t)\right] .
\end{aligned}
$$

Let

$$
N(u)=-I_{t}\left[\left(A(x, t) \frac{\partial}{\partial x}+B(x, t) \frac{\partial^{2}}{\partial x^{2}}\right) u(x, t)\right]
$$

As the above examples, we have the following first few components of the new iterative solution for the considered problem:

$$
\begin{gathered}
u_{0}(x, t)=1+x, \\
u_{1}(x, t)=(1+x) \cdot t, \\
u_{2}(x, t)=(1+x) \cdot \frac{t^{2}}{2}, \\
u_{3}(x, t)=(1+x) \cdot \frac{t^{3}}{6},
\end{gathered}
$$

and so on. The $(r+1)$-term approximate solution for this problem, in series form, is given by

$$
\begin{aligned}
u(x, t) & =\sum_{i=0}^{r} u_{i}(x, t) \\
& =(1+x) \cdot\left(1+t+\frac{t^{2}}{2 !}+\frac{t^{3}}{3 !}+\cdots\right),
\end{aligned}
$$

which in closed form gives the exact solution:

$$
u(x, t)=(1+x) \cdot e^{t} .
$$

By the IIM, according to (20), the initial value problem ((4) and (42)) is equivalent to the integral equation:

$$
\begin{aligned}
u_{r+1}(x, t) & \\
= & u_{0}(x, t) \\
& -I_{t}\left[\left(A(x, t) \frac{\partial}{\partial x}+B(x, t) \frac{\partial^{2}}{\partial x^{2}}\right) u_{r}(x, t)\right], \\
r & \geq 0 .
\end{aligned}
$$

As the above examples, we can have the following first few components of the integral iterative solution for the considered problem:

$$
\begin{gathered}
u_{0}(x, t)=1+x, \\
u_{1}(x, t)=(1+x) \cdot(1+t), \\
u_{2}(x, t)=(1+x) \cdot\left(1+t+\frac{t^{2}}{2}\right), \\
u_{3}(x, t)=(1+x) \cdot\left(1+t+\frac{t^{2}}{2}+\frac{t^{3}}{6}\right),
\end{gathered}
$$

and so on. In the same manner, the rest of components can be obtained. Hence,

$$
u_{r}(x, t)=(1+x) \cdot\left(1+t+\frac{t^{2}}{2 !}+\frac{t^{3}}{3 !}+\cdots+\frac{t^{r}}{r !}\right) .
$$


In closed form, this gives

$$
u(x, t)=\lim _{r \longrightarrow \infty} u_{r}=(1+x) \cdot e^{t},
$$

which is the same result as that obtained by the NIM in (48) and by both ADM and VIM in [2,7]. It is clear that the present two methods simplify the computational procedures and hence reduce the computational time more than the other methods. Therefore, IIM and NIM are better than ADM and VIM.

4.4. Example 4. Consider (5) with the initial condition

$$
u(x, 0)=x_{1}, \quad x=\left(x_{1}, x_{2}\right)^{T} \in R^{2} .
$$

Also, let

$$
\begin{aligned}
& A_{1}\left(x_{1}, x_{2}\right)=x_{1}, \\
& A_{2}\left(x_{1}, x_{2}\right)=5 x_{2}, \\
& B_{1,1}\left(x_{1}, x_{2}\right)=x_{1}^{2}, \\
& B_{1,2}\left(x_{1}, x_{2}\right)=1, \\
& B_{2,1}\left(x_{1}, x_{2}\right)=1, \\
& B_{2,2}\left(x_{1}, x_{2}\right)=x_{2}^{2} .
\end{aligned}
$$

By the NIM, the considered problem is equivalent to the integral equation:

$$
\begin{array}{r}
u(x, t)=x_{1}+I_{t}\left[\left(-\sum_{i=1}^{2} \frac{\partial}{\partial x_{i}} A_{i}\left(x_{1}, x_{2}\right)\right.\right. \\
\left.\left.+\sum_{i, j=1}^{2} \frac{\partial^{2}}{\partial x_{i} \partial x_{j}} B_{i, j}\left(x_{1}, x_{2}\right)\right) u(x, t)\right] .
\end{array}
$$

Let

$$
\begin{aligned}
& N(u)=I_{t}\left[\left(-\sum_{i=1}^{2} \frac{\partial}{\partial x_{i}} A_{i}\left(x_{1}, x_{2}\right)\right.\right. \\
& \left.\left.\quad+\sum_{i, j=1}^{2} \frac{\partial^{2}}{\partial x_{i} \partial x_{j}} B_{i, j}\left(x_{1}, x_{2}\right)\right) u(x, t)\right] .
\end{aligned}
$$

As the above examples, we have the following successive approximations:

$$
\begin{gathered}
u_{0}(x, t)=x_{1}, \\
u_{1}(x, t)=x_{1} \cdot t, \\
u_{2}(x, t)=x_{1} \cdot \frac{t^{2}}{2}, \\
u_{3}(x, t)=x_{1} \cdot \frac{t^{3}}{6},
\end{gathered}
$$

and so on. In closed form, the $(r+1)$-term approximate solution is given by

$$
u(x, t)=\sum_{i=1}^{r} u_{i}(x, t)=x_{1} \cdot\left(1+t+\frac{t^{2}}{2}+\frac{t^{3}}{6}+\cdots\right) .
$$

In closed form, this gives the exact solution:

$$
u(x, t)=x_{1} \cdot e^{t} .
$$

By the IIM, the considered problem is equivalent to the integral equation:

$$
\begin{gathered}
u_{r+1}(x, t)=u_{0}(x, t)+I_{t}\left[\left(-\sum_{i=1}^{2} \frac{\partial}{\partial x_{i}} A_{i}\left(x_{1}, x_{2}\right)\right.\right. \\
\left.\left.+\sum_{i, j=1}^{2} \frac{\partial^{2}}{\partial x_{i} \partial x_{j}} B_{i, j}\left(x_{1}, x_{2}\right)\right) u_{r}(x, t)\right], \quad r \geq 0 .
\end{gathered}
$$

Also, as above, we have the following successive approximations:

$$
\begin{gathered}
u_{0}(x, t)=x_{1}, \\
u_{1}(x, t)=x_{1} \cdot(1+t), \\
u_{2}(x, t)=x_{1} \cdot\left(1+t+\frac{t^{2}}{2}\right), \\
u_{3}(x, t)=x_{1} \cdot\left(1+t+\frac{t^{2}}{2}+\frac{t^{3}}{6}\right),
\end{gathered}
$$

and so on. In the same manner, the rest of components can be obtained. Hence,

$$
u_{r}(x, t)=x_{1} \cdot\left(1+t+\frac{t^{2}}{2}+\frac{t^{3}}{6}+\cdots+\frac{t^{r}}{r !}\right)
$$

In closed form, this gives

$$
u(x, t)=\lim _{r \rightarrow \infty} u_{r}=x_{1} \cdot e^{t},
$$

which is the same result as that obtained by the NIM in (59) and by both ADM and VIM in $[2,7]$. It is clear that the present two methods simplify the computational procedures and hence reduce the computational time more than the other methods. Therefore, IIM and NIM are better than ADM and VIM.

4.5. Example 5. Consider the nonlinear FPE (7), such that

$$
\begin{aligned}
u(x, 0) & =x^{2}, \quad x \in \mathrm{Re}, \\
A(x, t, u) & =\frac{4}{x} u-\frac{x}{3}, \\
B(x, t, u) & =u .
\end{aligned}
$$


By the NIM, this problem is equivalent to the integral equation:

$$
\begin{aligned}
& u(x, t) \\
& =x^{2} \\
& \quad+I_{t}\left[\left(-\frac{\partial}{\partial x} A(x, t, u)+\frac{\partial^{2}}{\partial x^{2}} B(x, t, u)\right) u(x, t)\right] .
\end{aligned}
$$

Let

$$
\begin{aligned}
& N(u) \\
& \quad=I_{t}\left[\left(-\frac{\partial}{\partial x} A(x, t, u)+\frac{\partial^{2}}{\partial x^{2}} B(x, t, u)\right) u(x, t)\right] .
\end{aligned}
$$

In view of (13), we can have the following successive approximations:

$$
\begin{gathered}
u_{0}(x, t)=x^{2}, \\
u_{1}(x, t)=x^{2} \cdot t, \\
u_{2}(x, t)=x^{2} \cdot \frac{t^{2}}{2}, \\
u_{3}(x, t)=x^{2} \cdot \frac{t^{3}}{6},
\end{gathered}
$$

and so on. The $(r+1)$-term new iterative approximate solution for this problem takes the form

$$
u(x, t)=\sum_{i=0}^{r} u_{i}=x^{2} \cdot\left(1+t+\frac{t^{2}}{2 !}+\frac{t^{3}}{3 !}+\cdots\right),
$$

which in closed form gives the exact solution:

$$
u(x, t)=x^{2} \cdot e^{t} .
$$

By the IIM, the initial value problem $((7)$ and (64)) is equivalent to the integral equation:

$$
\begin{array}{r}
u_{r+1}(x, t)=u_{0}(x, t) \\
+I_{t}\left[\left(-\frac{\partial}{\partial x} A(x, t, u)+\frac{\partial^{2}}{\partial x^{2}} B(x, t, u)\right) u_{r}(x, t)\right] \\
r \geq 0 .
\end{array}
$$

Also, as above, we can have the following successive approximations:

$$
\begin{gathered}
u_{0}(x, t)=x^{2}, \\
u_{1}(x, t)=x^{2} \cdot(1+t), \\
u_{2}(x, t)=x^{2} \cdot\left(1+t+\frac{t^{2}}{2}\right), \\
u_{3}(x, t)=x^{2} \cdot\left(1+t+\frac{t^{2}}{2}+\frac{t^{3}}{6}\right),
\end{gathered}
$$

and so on. In the same manner, the rest of components can be obtained. Hence,

$$
u_{r}(x, t)=x^{2} \cdot\left(1+t+\frac{t^{2}}{2 !}+\frac{t^{3}}{3 !}+\cdots+\frac{t^{r}}{r !}\right)
$$

and, in closed form, this gives

$$
u(x, t)=\lim _{r \longrightarrow \infty} u_{r}=x^{2} \cdot e^{t}
$$

which is the same result as that obtained by NIM in (69) and by both ADM and VIM in [2,7]. It is clear that the present two methods simplify the computational procedures and hence reduce the computational time more than the other methods. Therefore, IIM and NIM are better than ADM and VIM.

4.6. Example 6. Finally, consider the generalized nonlinear FPE (8), such that

$$
\begin{aligned}
u(x, 0) & =x_{1}^{2}, \quad x=\left(x_{1}, x_{2}\right)^{T} \in R^{2}, \\
A_{1}(x, t, u) & =\frac{4}{x_{1}} u, \\
A_{2}(x, t, u) & =x_{2}, \\
B_{1,1}\left(x_{1}, x_{2}\right) & =u, \\
B_{1,2}\left(x_{1}, x_{2}\right) & =1, \\
B_{2,1}\left(x_{1}, x_{2}\right) & =1, \\
B_{2,2}\left(x_{1}, x_{2}\right) & =u .
\end{aligned}
$$

By the NIM, the problem in (8) and (75) is equivalent to the integral equation:

$$
\begin{array}{r}
u(x, t)=x_{1}^{2}+I_{t}\left[\left(-\sum_{i=1}^{2} \frac{\partial}{\partial x_{i}} A_{i}(x, t, u)\right.\right. \\
\left.\left.+\sum_{i, j=1}^{2} \frac{\partial^{2}}{\partial x_{i} \partial x_{j}} B_{i, j}(x, t, u)\right) u(x, t)\right] .
\end{array}
$$

Let

$$
\begin{aligned}
& N(u)=I_{t}\left[\left(-\sum_{i=1}^{2} \frac{\partial}{\partial x_{i}} A_{i}(x, t, u)\right.\right. \\
& \left.\left.\quad+\sum_{i, j=1}^{2} \frac{\partial^{2}}{\partial x_{i} \partial x_{j}} B_{i, j}(x, t, u)\right) u(x, t)\right] .
\end{aligned}
$$


In view of (13), we have the following set of successive approximations for this problem:

$$
\begin{gathered}
u_{0}(x, t)=x_{1}^{2}, \\
u_{1}(x, t)=-x_{1}^{2} \cdot t, \\
u_{2}(x, t)=x_{1}^{2} \cdot \frac{t^{2}}{2}, \\
u_{3}(x, t)=-x_{1}^{2} \cdot \frac{t^{3}}{6},
\end{gathered}
$$

The $(r+1)$-term approximate solution for this problem is

$$
u(x, t)=\sum_{i=0}^{r} u_{i}=x_{1}^{2} \cdot\left(1-t+\frac{t^{2}}{2 !}-\frac{t^{3}}{3 !}+\cdots\right)
$$

which in closed form gives the exact solution:

$$
u(x, t)=x_{1}^{2} \cdot e^{-t}
$$

By the IIM, the problem in (8) and (75) is equivalent to the integral equation:

$$
\begin{gathered}
u_{r+1}(x, t)=u_{0}(x, t)+I_{t}\left[\left(-\sum_{i=1}^{2} \frac{\partial}{\partial x_{i}} A_{i}(x, t, u)\right.\right. \\
\left.\left.+\sum_{i, j=1}^{2} \frac{\partial^{2}}{\partial x_{i} \partial x_{j}} B_{i, j}(x, t, u)\right) u_{r}(x, t)\right], \quad r \geq 0 .
\end{gathered}
$$

As above, we have the following first few components of the integral iterative solution for (8) and (75):

$$
\begin{gathered}
u_{0}(x, t)=x_{1}^{2}, \\
u_{1}(x, t)=x_{1}^{2} \cdot(1-t), \\
u_{2}(x, t)=x_{1}^{2} \cdot\left(1-t+\frac{t^{2}}{2}\right), \\
u_{3}(x, t)=x_{1}^{2} \cdot\left(1-t+\frac{t^{2}}{2}-\frac{t^{3}}{6}\right),
\end{gathered}
$$

and so on. In the same manner, the rest of components can be obtained. Hence,

$$
u_{r}(x, t)=x_{1}^{2} \cdot\left(1-t+\frac{t^{2}}{2 !}-\frac{t^{3}}{3 !}+\cdots(-1)^{r} \frac{t^{r}}{r !}\right) .
$$

In closed form, this gives

$$
u(x, t)=\lim _{r \longrightarrow \infty} u_{r}=x_{1}^{2} \cdot e^{-t}
$$

which is the same result as that obtained by the NIM in (80) and by both the ADM and VIM in $[2,7]$. It is clear that the present two methods simplify the computational procedures and hence reduce the computational time more than the other methods. Therefore, IIM and NIM are better than ADM and VIM.

\section{Conclusion}

In this work, both NIM and IIM have been applied successfully for solving Fokker-Planck equation. The obtained results are compared with those obtained by both ADM and VIM. It is apparently seen that NIM, IIM, VIM, and ADM are very efficient and powerful to get the exact solutions in a rapid convergent form. The comparison of the results shows that although the results of the four methods are the same, NIM and IIM provide the solutions of the solved problems without using Adomian's polynomials or Lagrange's multipliers, which can be considered as an advantage of both NIM and IIM over both ADM and VIM. Therefore, NIM and IIM are easier and so more convenient than ADM and VIM.

\section{Data Availability}

No data were used to support this study.

\section{Conflicts of Interest}

The authors declare that there are no conflicts of interest regarding the publication of this paper.

\section{References}

[1] N. Dogan, "Solution of the system of ordinary differential equations by combined Laplace transform-Adomian decomposition method," Mathematical \& Computational Applications, vol. 17, no. 3, pp. 203-211, 2012.

[2] M. Tatari, M. Dehghan, and M. Razzaghi, "Application of the Adomian decomposition method for the Fokker-Planck equation," Mathematical and Computer Modelling, vol. 45, no. 5-6, pp. 639-650, 2007.

[3] A. A. Hemeda, "Variational iteration method for solving nonlinear partial differential equations," Chaos, Solitons \& Fractals, vol. 39, no. 3, pp. 1297-1303, 2009.

[4] A. A. Hemeda, "Variational iteration method for solving nonlinear coupled equations in 2-dimensional space in fluid mechanics," International Journal of Contemporary Mathematical Sciences, vol. 7, no. 37, pp. 1839-1852, 2012.

[5] A. F. Elsayed, "Comparison between variational iteration method and homotopy perturbation method for thermal diffusion and diffusion thermo effects of thixotropic fluid through biological tissues with laser radiation existence," Applied Mathematical Modelling: Simulation and Computation for Engineering and Environmental Systems, vol. 37, no. 6, pp. 3660-3673, 2013.

[6] A. A. Elbeleze, A. Kiliçman, and B. M. Taib, "Application of Homotopy Perturbation and Variational Iteration Methods for Fredholm Integrodifferential Equation of Fractional Order," Abstract and Applied Analysis, vol. 2012, Article ID 763139, 14 pages, 2012. 
[7] J. Biazar, P. Gholamin, and K. Hosseini, "Variational iteration method for solving Fokker-Planck equation," Journal of The Franklin Institute, vol. 347, no. 7, pp. 1137-1147, 2010.

[8] S. X. Liang and D. J. Jeffrey, "Comparison of homotopy analysis method and homotopy perturbation method through an evolution equation," Communications in Nonlinear Science and Numerical Simulation, vol. 14, no. 12, pp. 4057-4064, 2009.

[9] M. A. Asadi, F. Salehi, S. T. M. Din, and M. M. Hosseini, "Modified homotopy perturbation method for stiff delay differential equations (DDEs)," International Journal of Physical Sciences, vol. 7, no. 7, pp. 1025-1034, 2012.

[10] B. Ghazanfari, A. Ghazanfari, and M. Fuladvand, "Modification Of The Homotopy Perturbation Method For Numerical Solution Of Nonlinear Wave And System Of Nonlinear Wave Equations," Journal of Mathematics and Computer Science, vol. 03, no. 02, pp. 212-224, 2011.

[11] Y. Niu, Z. Wang, and S. Dong, "Modified Homotopy Perturbation Method for Certain Strongly Nonlinear Oscillators," Chinese Physics Letters, vol. 29, no. 6, pp. 060502/1-060502/4, 2012.

[12] S. K. Vanani and F. Soleymani, "Application of the Homotopy Perturbation Method to the Burgers Equation with Delay," Chinese Physics Letters, vol. 29, no. 3, pp. 030202/1-030202/4, 2012.

[13] J. Biazar, K. Hosseini, and P. Gholamin, "Homotopy perturbation method Fokker-Planck equation," International Mathematical Forum. Journal for Theory and Applications, vol. 3, no. 17-20, pp. 945-954, 2008.

[14] H. A. Zedan and E. El Adrous, "The Application of the Homotopy Perturbation Method and theHomotopy Analysis Method to the Generalized Zakharov Equations," Abstract and Applied Analysis, vol. 2012, Article ID 561252, 19 pages, 2012.

[15] V. Daftardar-Gejji and H. Jafari, "An iterative method for solving nonlinear functional equations," Journal of Mathematical Anal$y$ sis and Applications, vol. 316, no. 2, pp. 753-763, 2006.

[16] S. Bhalekar and V. Daftardar-Gejji, "New iterative method: application to partial differential equations," Applied Mathematics and Computation, vol. 203, no. 2, pp. 778-783, 2008.

[17] A. A. Hemeda, "New Iterative Method: An Application for Solving Fractional Physical Differential Equations," Abstract and Applied Analysis, vol. 2013, Article ID 617010, 9 pages, 2013.

[18] V. Daftardar-Gejji and S. Bhalekar, "Solving fractional diffusion-wave equations using a new iterative method," Fractional Calculus \& Applied Analysis. An International Journal for Theory and Applications, vol. 11, no. 2, pp. 193-202, 2008.

[19] V. Daftardar-Gejji and S. Bhalekar, "Solving fractional boundary value problems with Dirichlet boundary conditions using a new iterative method," Computers \& Mathematics with Applications, vol. 59, no. 5, pp. 1801-1809, 2010.

[20] V. Daftardar-Gejji and S. Bhalekar, "An iterative method for solving fractional differential equations," Proceeding in Applied Mathematics and Mathematics, vol. 1, no. 7, pp. 20500172050018, 2008.

[21] H. Jafari, S. Seifi, A. Alipoor, and M. Zabihi, "An iterative method for solving linear and nonlinear fractional diffusionwave equation," International eJournal of Numerical Analysis \& Related Topics, vol. 3, pp. 20-32, 2009.

[22] M. Thongmoon and S. Pusjuso, "The numerical solutions of differential transform method and the Laplace transform method for a system of differential equations," Nonlinear Analysis: Hybrid Systems, vol. 4, no. 3, pp. 425-431, 2010.
[23] A. A. Hemeda, "An integral iterative method for solving fractional physical differential equations," INFORMATION-An International Interdisciplinary Journal, vol. 18, no. 2, pp. 365-381, 2015.

[24] A. A. Hemeda, "A friendly iterative technique for solving nonlinear integro-differential and systems of nonlinear integrodifferential equations," International Journal of Computational Methods, vol. 15, no. 3, 1850016, 15 pages, 2018.

[25] J. He, "Some asymptotic methods for strongly nonlinear equations," International Journal of Modern Physics B, vol. 20, no. 10, pp. 1141-1199, 2006.

[26] M. A. Noor, K. I. Noor, E. Al-Said, and M. Waseem, "Some New Iterative Methods for Nonlinear Equations," Mathematical Problems in Engineering, vol. 2010, Article ID 198943, 12 pages, 2010.

[27] S. T. Mohyud-Din, A. Yildirim, and S. M. Hosseini, "Numerical comparison of methods for Hirota-Satsuma model," Applications and Applied Mathematics. An International Journal, vol. 5, no. 10, pp. 1554-1563, 2010.

[28] H. Risken, The FokkerPlanck Equation: Method of Solution and Applications, Springer, Berlin, Germany, 1989.

[29] T. D. Frank, "Stochastic feedback, nonlinear families of Markov processes, and nonlinear Fokker-Planck equations," Physica A: Statistical Mechanics and its Applications, vol. 331, no. 3-4, pp. 391-408, 2004.

[30] Y. Cherruault, “Convergence of Adomian's method," Kybernetes, vol. 18, no. 2, pp. 31-38, 1989.

[31] A. J. Jerri, Introduction to Integral Equations with Applications, John Wiley \& Sons, New York, NY, USA, 2nd edition, 1999.

[32] A. A. Hemeda, E. E. Eladdad, and I. A. Lairje, "Local fractional analytical methods for solving wave equations with local fractional derivative," Mathematical Methods in the Applied Sciences, vol. 41, no. 6, pp. 1-15, 2018. 


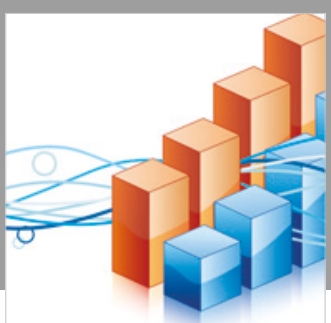

Advances in

Operations Research

\section{-n-m}
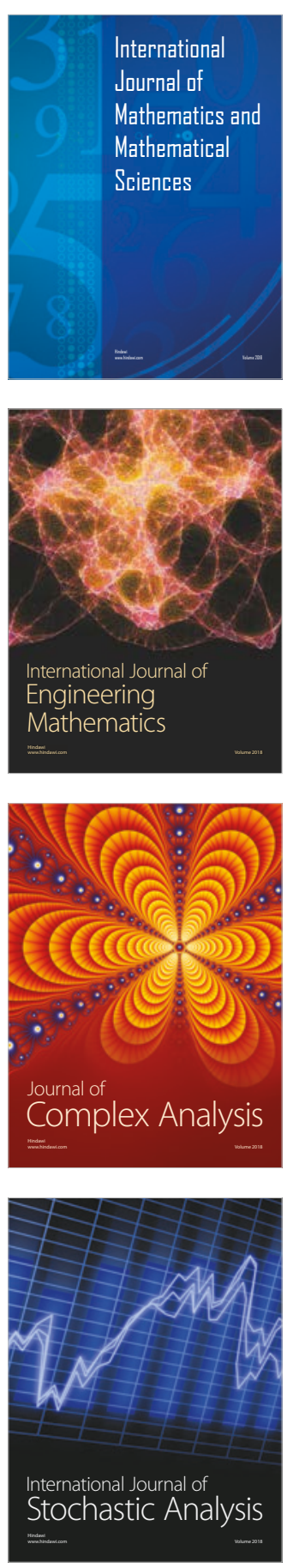
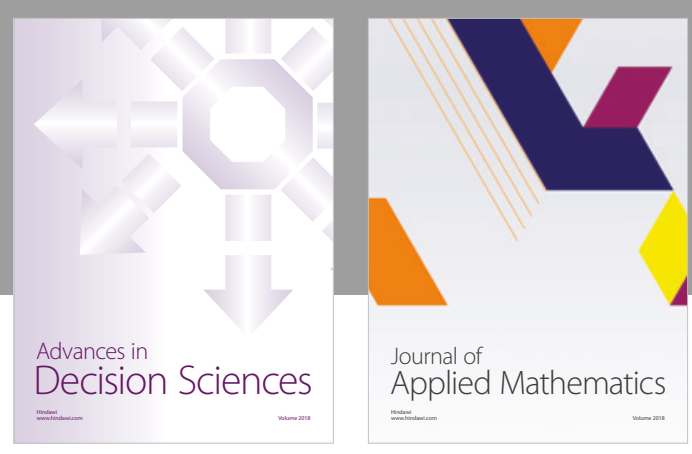

Journal of

Applied Mathematics
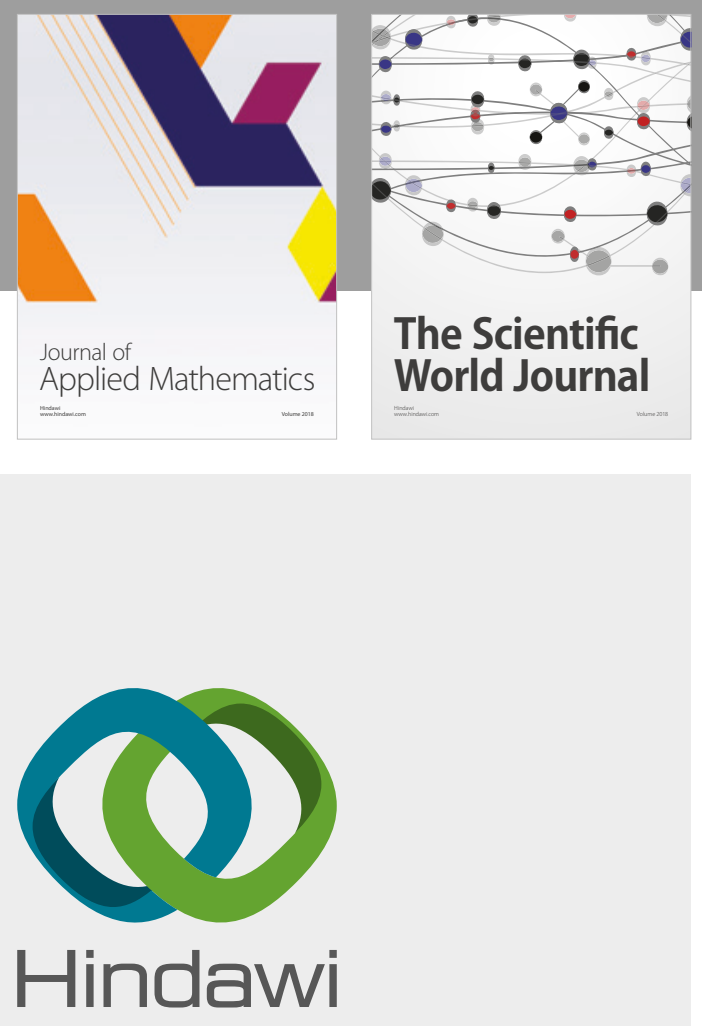

Submit your manuscripts at

www.hindawi.com

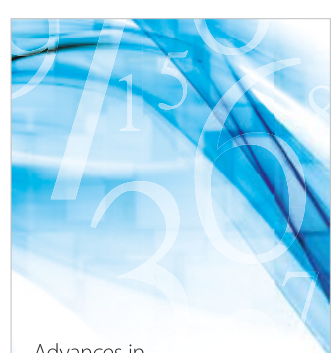

Advances in
Numerical Analysis
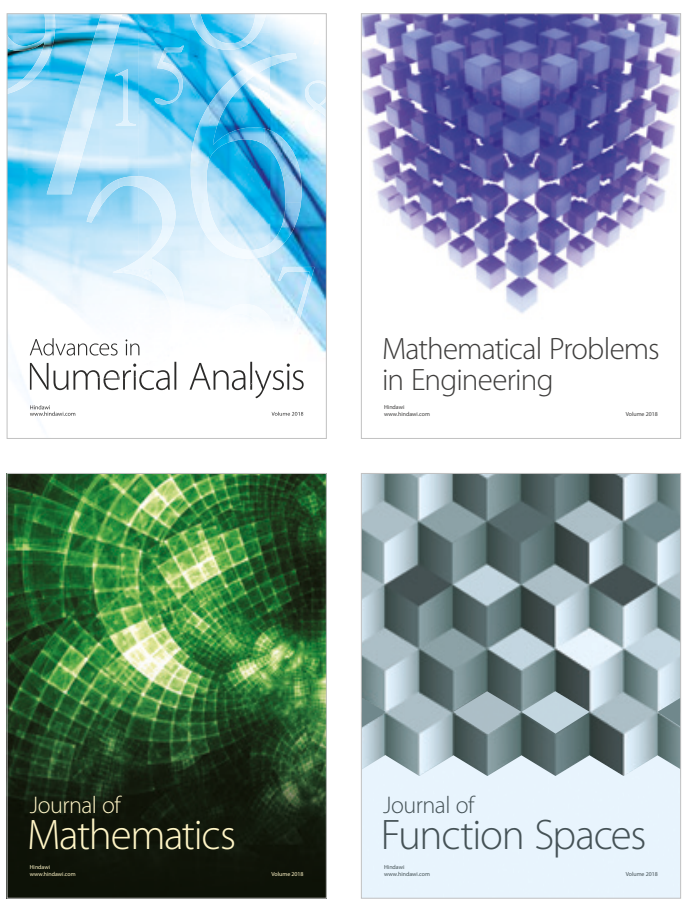

Mathematical Problems in Engineering

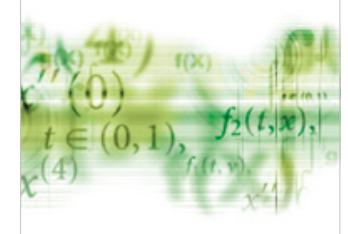

International Journal of

Differential Equations

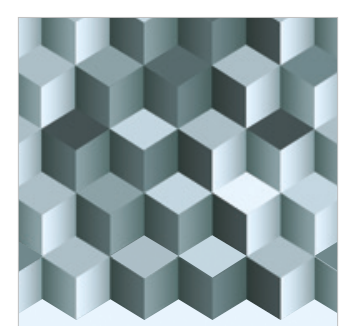

Journal of

Function Spaces

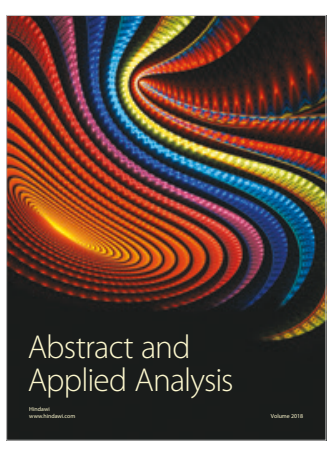

The Scientific

World Journal

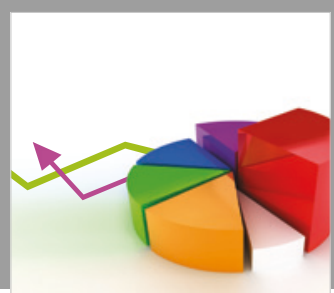

Journal of

Probability and Statistics
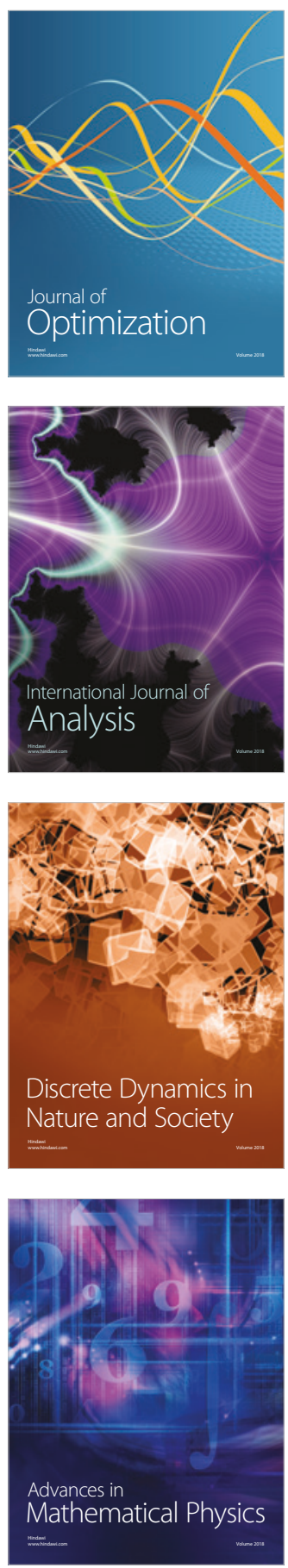